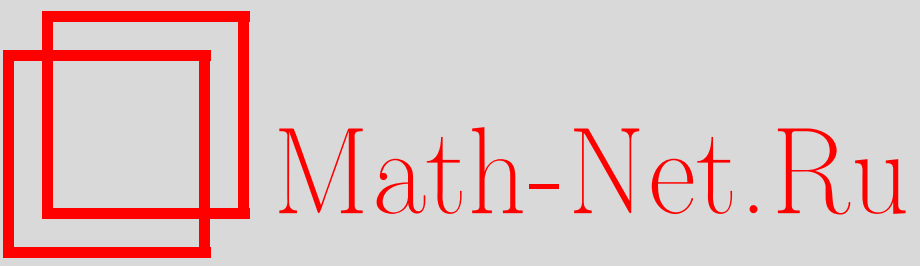

С. А. Хихол, Формула для обобщенного процесса плотности распределений семимартингалов с независимыми приращениями, Теория вероятн. и ее примен., 2009, том 54, выпуск 4, 716-729

DOI: https://doi.org/10.4213/tvp3536

Использование Общероссийского математического портала Math-Net.Ru подразумевает, что вы прочитали и согласны с пользовательским соглашением

http: //www . mathnet.ru/rus/agreement

Параметры загрузки:

IP : 54.147 .182 .235

26 апреля 2023 г., 17:36:26 
2009

(c) 2009 г.

ХИХОЛ С. А.*

\title{
ФОРМУЛА ДЛЯ ОБОБЩЕННОГО ПРОЦЕССА ПЛОТНОСТИ РАСПРЕДЕЛЕНИЙ СЕМИМАРТИНГАЛОВ С НЕЗАВИСИМЫМИ ПРИРАЩЕНИЯМИ
}

\begin{abstract}
В статье доказывается формула для процесса плотности распределений двух семимартингалов с независимыми приращениями, обобщающая хорошо известный результат, относящийся к случаю локально абсолютно непрерывных распределений, а также результат Сато для процессов Леви без требования локальной абсолютной непрерывности.
\end{abstract}

Ключевые слова и фразы: семимартингал, процесс с независимыми приращениями, процесс плотности, триплет локальных характеристик.

1. Формулировка результата. Для двух семимартингалов с независимыми приращениями хорошо известно выражение для процесса плотности их распределений в случае, когда одно из них локально абсолютно непрерывно относительно другого [1, теорема III.5.35]. Известна также формула для обобщенного процесса плотности распределений процессов Леви без требования локальной абсолютной непрерывности (см. [2, формула (3.32)]). Цель данной работы — получить формулу для (обобщенного) процесса плотности распределений двух произвольных семимартингалов с независимыми приращениями. Термины и обозначения, не объясняемые ниже, следуют монографии Жакода, Ширяева [1].

Пусть $\Omega=\mathbf{D}\left(\mathbf{R}^{d}\right)=\mathbf{D}\left(\mathbf{R}_{+} ; \mathbf{R}^{d}\right)$ - пространство всех непрерывных справа, имеющих пределы слева функций $\omega: \mathbf{R}_{+} \rightarrow \mathbf{R}^{d}, X-$ канонический процесс, задаваемый соотношением $X_{t}(\omega)=\omega(t), \mu$ - мера скачков процесса $X, \mathscr{H}=\sigma\left(X_{0}\right)$, фильтрация $\mathbf{F}=\left(\mathscr{F}_{t}\right)_{t \in \mathbf{R}_{+}}$порождена $X$, т.е. $\mathscr{F}_{t}=\bigcap_{\varepsilon>0} \mathscr{F}_{t+\varepsilon}^{0}, \mathscr{F}_{t}^{0}=\sigma\left\{X_{s}, s \leqslant t\right\}, \mathscr{F}=\mathscr{F}_{\infty}=\mathscr{F}_{\infty}^{0}$. Для произвольной меры $\mathrm{Q}$ будем обозначать $\mathrm{Q}_{t}$ и $\mathrm{Q}_{t}^{0}$ ее сужения на $\sigma$-алгебры $\mathscr{F}_{t}$ и $\mathscr{F}_{t}^{0}$ соответственно.

Предположим, что на $(\Omega, \mathscr{F})$ заданы две вероятностные меры $\mathrm{P}$ и $\mathrm{P}^{\prime}$, по которым процесс $X-X_{0}$ является семимартингалом с независимыми приращениями. Под обобщенным прочессом плотности меры $\mathrm{P}^{\prime}$

* Московский государственный университет им. М.В.Ломоносова, механикоматематический факультет, Ленинские горы 1, 119991 Москва, Россия; e-mail: sahihol@gmail.com 
относительно P будем понимать такой (единственный с точностью до Pнеразличимости) согласованный $\mathrm{P}$-п.н. непрерывный справа и имеющий пределы слева случайный процесс $Z=\left(Z_{t}\right)_{t \in \mathbf{R}_{+}}$со значениями в $\mathbf{R}_{+}$, что для любого $t \in \mathbf{R}_{+}$случайная величина $Z_{t}$ есть плотность абсолютно непрерывной компоненты $\mathrm{P}_{t}^{\prime}$ относительно $\mathrm{P}_{t}$. Напомним, что если $\mathrm{P}$ и $\mathrm{P}^{\prime}$ локально абсолютно непрерывны относительно вероятностной меры $\mathrm{Q}$, а $z$ и $z^{\prime}-$ соответствующие процессы плотности, то $\mathrm{P}\left(\inf _{t} z_{t}=0\right)=0$ и процессы $Z$ и $z^{\prime} / z$ Р-неразличимы. В частности, обобщенный процесс плотности совпадает с обычным процессом плотности, если $\mathrm{P}^{\prime}$ локально абсолютно непрерывна относительно $\mathrm{P}$.

Фиксируем функцию урезания $\hbar: \mathbf{R}^{d} \rightarrow \mathbf{R}^{d}$. Хорошо известно, что триплеты характеристик процесса $X$ относительно обеих мер можно выбрать детерминированными. Обозначим эти триплеты соответственно

$$
\left(B_{t}, C_{t}, \nu(d t, d x)\right), \quad\left(B_{t}^{\prime}, C_{t}^{\prime}, \nu^{\prime}(d t, d x)\right) .
$$

Обозначим также $\mathrm{P}_{H}=\mathrm{P}_{0}^{0}$ и $\mathrm{P}_{H}^{\prime}=\mathrm{P}_{0}^{\prime 0}$. Свяжем с триплетами (1) несколько детерминированных множеств и процессов. Во-первых, положим

$$
\begin{aligned}
& \nu_{t}(d x)=\nu(\{t\} \times d x), \quad a_{t}=\nu_{t}\left(\mathbf{R}^{d}\right), \quad J=\left\{t: a_{t}>0\right\} \\
& \kappa_{t}(d x)=\nu_{t}(d x)+\left(1-a_{t}\right) \delta_{\{0\}}(d x), \\
& \nu_{t}^{\prime}(d x)=\nu^{\prime}(\{t\} \times d x), \quad a_{t}^{\prime}=\nu_{t}^{\prime}\left(\mathbf{R}^{d}\right), \quad J^{\prime}=\left\{t: a_{t}^{\prime}>0\right\}, \\
& \kappa_{t}^{\prime}(d x)=\nu_{t}^{\prime}(d x)+\left(1-a_{t}^{\prime}\right) \delta_{\{0\}}(d x) .
\end{aligned}
$$

Напомним, что меры $\nu$ и $\nu^{\prime}$ не нагружают множества $\{0\} \times \mathbf{R}^{d}$ и $\mathbf{R}_{+} \times\{0\}$, $\Delta B_{t}=\int \hbar(x) \nu(\{t\} \times d x), \Delta B_{t}^{\prime}=\int \hbar(x) \nu^{\prime}(\{t\} \times d x)$. Далее, существует непрерывная возрастающая функция $A_{t}$ и две функции $c_{t}, c_{t}^{\prime}$ со значениями во множестве симметрических неотрицательно определенных матриц размера $d \times d$ такие, что $C=c \cdot A, C^{\prime}=c^{\prime} \cdot A$.

Пусть $\lambda$ - мера на $\mathbf{R}_{+} \times \mathbf{R}^{d}$ такая, что $\left(|x|^{2} \wedge 1\right) * \lambda_{t}<\infty$ для всех $t<\infty$ и $\nu \ll \lambda, \nu^{\prime} \ll \lambda$. Положим $U=d \nu / d \lambda, U^{\prime}=d \nu^{\prime} / d \lambda$.

Пусть $\Sigma=\left\{t:\left|\hbar(x)\left(U-U^{\prime}\right)\right| * \lambda_{t}<\infty\right\}$. На множестве $\Sigma$ существует разложение

$$
B^{\prime}=B+\hbar(x)\left(U^{\prime}-U\right) * \lambda+(c \beta) \cdot A+\beta^{\perp} \cdot A+\widetilde{B},
$$

где $\beta$ и $\beta^{\perp}$ - измеримые функции $\Sigma \rightarrow \mathbf{R}^{d}, \widetilde{B}-$ функция $\Sigma \rightarrow \mathbf{R}^{d}$ с ограниченной вариацией на компактных интервалах, $d \widetilde{B}$ и $d A$ взаимно сингулярны на $\Sigma$ и для всех $t$ вектор $\beta_{t}^{\perp}$ ортогонален к образу $\mathbf{R}^{d}$ при линейном отображении, связанном с матрицей $c_{t}$. Существование разложения (2) (в более общей ситуации) обсуждается в [1, гл. IV, § 3a]. Как мы увидим далее, если меры $\mathrm{P}_{t}$ и $\mathrm{P}_{t}^{\prime}$ несингулярны для всех $t \in \mathbf{R}_{+}$, то $\Sigma=\mathbf{R}_{+}$и два последних члена в разложении (2) отсутствуют. 
Рассмотрим детерминированный момент $\sigma$ :

$$
\sigma=\min _{i=1, \ldots, 6} \sigma_{i}
$$

где

$$
\begin{aligned}
& \sigma_{1}=\left\{\begin{array}{l}
0, \text { если } \mathrm{P}_{H} \perp \mathrm{P}_{H}^{\prime}, \\
\infty \text { иначе, }
\end{array} \quad \sigma_{2}=\inf \left\{t:\left|\hbar(x)\left(U-U^{\prime}\right)\right| * \lambda_{t}=\infty\right\},\right. \\
& \sigma_{3}=\inf \left\{t: C_{t} \neq C_{t}^{\prime}\right\}, \quad \sigma_{4}=\inf \left\{t \in \Sigma: \beta^{\perp} \cdot A_{t}+\widetilde{B}_{t} \neq 0\right\}, \\
& \sigma_{5}=\inf \left\{t \in \Sigma: h^{0}\left(\frac{1}{2}\right)_{t}=\infty\right\}, \quad \sigma_{6}=\inf \left\{t: \kappa_{t} \perp \kappa_{t}^{\prime}\right\}, \\
& h^{0}\left(\frac{1}{2}\right)_{t}=\frac{1}{8}\left(\beta^{T} c \beta\right) \cdot A_{t}+\frac{1}{2}\left(\sqrt{U}-\sqrt{U^{\prime}}\right)^{2} * \lambda_{t} \\
& +\frac{1}{2} \sum_{s \leqslant t}\left(\sqrt{1-a_{s}}-\sqrt{1-a_{s}^{\prime}}\right)^{2} \text {. }
\end{aligned}
$$

Как обычно, $\inf \varnothing=\infty$.

Наконец, существует единственное разложение $\nu^{\prime}=\nu_{1}^{\prime}+\nu_{2}^{\prime}$, где $\nu_{1}^{\prime} \ll \nu$ и $\nu_{2}^{\prime} \perp \nu$, причем $\nu_{1}^{\prime}=1_{\mathbf{A}} \cdot \nu^{\prime}$ и $\nu_{2}^{\prime}=1_{\mathbf{A}^{c}} \cdot \nu^{\prime}$ для некоторого множества $\mathbf{A} \in \mathscr{B}\left(\mathbf{R}_{+} \times \mathbf{R}^{d}\right)$. Обозначим $Y-$ вариант плотности $\nu_{1}^{\prime}$ относительно $\nu: \nu_{1}^{\prime}=Y \cdot \nu$. Например, можно взять $\mathbf{A}=\{U>0\}$ и $Y=\left(U^{\prime} / U\right) 1_{\mathbf{A}}$. Обозначим также $a_{i, t}^{\prime}=\nu_{i}^{\prime}\left(\{t\} \times \mathbf{R}^{d}\right), i=1,2$.

Мы можем теперь сформулировать основной результат статьи.

Теорема 1. Пусть прочесс $X-X_{0}$ является семимартингалом с независимыми приращениями относительно мер $\mathrm{P}$ и $\mathrm{P}^{\prime}$ с триплетами локальньх характеристик (1), и детерминированньий момент $\sigma$ задается соотношением (3). Тогда

$$
\sigma=\min \left\{t: \mathrm{P}_{t} \perp \mathrm{P}_{t}^{\prime}\right\},
$$

и обобщенныци прочесс плотности $Z$ мерь $\mathrm{P}^{\prime}$ относительно $\mathrm{P}$ имеет следующий вид: Р-п.н.

$$
Z=\frac{d \mathrm{P}_{H}^{\prime}}{d \mathrm{P}_{H}} \mathscr{E}(N) D 1_{\llbracket 0, \sigma \llbracket}=\frac{d \mathrm{P}_{H}^{\prime}}{d \mathrm{P}_{H}} \mathscr{E}(N-\Psi) 1_{\llbracket 0, \sigma \llbracket,},
$$

әде

$$
\begin{aligned}
& N_{t}=\beta \cdot X_{t}^{c}+\left(Y-1+\frac{a_{1}^{\prime}-a}{1-a} 1_{\{a<1\}}\right) *(\mu-\nu)_{t}, \\
& D_{t}=\exp \left(-k_{t}\right) \prod_{\substack{s \leqslant t, a_{s}<1, \Delta X_{s}=0, a_{1, s}^{\prime}<1}} \frac{1-a_{s}^{\prime}}{1-a_{1, s}^{\prime}}
\end{aligned}
$$




$$
\begin{gathered}
\times \prod_{\substack{s \leqslant t, a_{s}=1, \\
\text { unu } Y\left(s, \Delta X_{s}\right)>0}} \frac{Y\left(s, \Delta X_{s}\right)}{1+Y\left(s, \Delta X_{s}\right)-a_{1, s}^{\prime}}, \\
\Psi_{t}=k_{t}+\sum_{\substack{s \leqslant t, a_{s}<1, \Delta X_{s}=0}} \frac{a_{2, s}^{\prime}}{1-a_{s}}+\sum_{s \leqslant t, a_{s}=1}\left(1-a_{1, s}^{\prime}\right), \\
k_{t}=\nu_{2}^{\prime}\left(\left\{s \leqslant t: a_{s}=a_{s}^{\prime}=0\right\} \times \mathbf{R}^{d}\right) .
\end{gathered}
$$

3 а м е ч а н и е 1. Представление (5) неявно требует выполнения условий $\beta 1_{[0, t]} \in L_{l o c}^{2}\left(X^{c}, \mathrm{P}\right)$ и $\left(Y-1+\left(\left(a_{1}^{\prime}-a\right) /(1-a)\right) 1_{\{a<1\}}\right) 1_{[0, t]} \in$ $G_{l o c}(\mu, \mathrm{P})$ для любого $t<\sigma$. Первое произведение в определении $D$ всегда корректно определено, так как при $s \notin J^{\prime}$ множитель $\left(1-a_{s}^{\prime}\right) /\left(1-a_{1, s}^{\prime}\right)$ равен 1.

3 а м е ч а н и е 2. Несложно видеть, что определение момента $\sigma$ и представление (5) не зависят от выбора меры $\lambda$, функций $A, c, c^{\prime}, \beta, Y$.

В случае $\mathrm{P}^{\prime} \stackrel{\text { loc }}{\ll} \mathrm{P}$, согласно критерию абсолютной непрерывности $[1$, теорема IV.4.32], имеем $\sigma=\infty, \nu^{\prime} \ll \nu$ и $a_{t}^{\prime}=1$, если $a_{t}=1$, поэтому $D \equiv 1, \Psi \equiv 0$ и формула (5) сводится к известной формуле [1, III.5.21] для процесса плотности.

$\mathrm{B}$ случае, когда $\mathrm{P}$ и $\mathrm{P}^{\prime}-$ распределения процессов Леви, известно (и следует из теоремы 1 ), что $\sigma=0$ или $\sigma=\infty$. Поскольку $a_{t} \equiv a_{t}^{\prime} \equiv 0$, в случае $\sigma=\infty$ представление (5) приобретает вид

$$
Z=\mathscr{E}\left(\beta \cdot X^{c}+(Y-1) *(\mu-\nu)-k\right),
$$

причем $\beta$ не зависит от $t$, а $Y$ зависит только от $x$. Несложно проверить, что это представление для $Z$ совпадает с несколько более сложным представлением $[2,(3.32)]$ у Сато.

Приведем несколько примеров, в которых процесс $D$ отличен от единицы.

П р и м е р 1. Пусть $X \equiv 0$ по мере Р и $X-$ пуассоновский процесс с интенсивностью $\lambda$ по мере $\mathrm{P}^{\prime}$. Очевидно, что обобщенный процесс плотности $Z_{t}$ удовлетворяет соотношению $Z_{t}=\mathrm{P}^{\prime}\left(X_{t}=0\right)=\exp (-\lambda t)$ Р-п.н. Тот же ответ дает формула (5): ясно, что Р-п.н. $d \mathrm{P}_{H}^{\prime} / d \mathrm{P}_{H}=1$, $\sigma=\infty, N \equiv 0, k_{t}=\lambda t, D_{t}=\exp \left(-k_{t}\right)$.

П р и м е р 2. Предположим, что Р- и $\mathrm{P}^{\prime}$-п.н. $X_{t}=0$ для любого $t<1$ и $X_{t}=X_{1}$ для любого $t>1$. Обозначим $\mathrm{Q}(B)=\mathrm{P}\left(\Delta X_{1} \in B\right)$, $\mathrm{Q}^{\prime}(B)=\mathrm{P}^{\prime}\left(\Delta X_{1} \in B\right)$, где $B \in \mathscr{B}\left(\mathbf{R}^{d}\right)$. Ясно, что версия процесса $Z_{t}$ задается равенством

$$
Z_{t}=\left\{\begin{array}{l}
1 \text { при } t<1, \\
\frac{d \mathbf{Q}^{\prime c}}{d \mathrm{Q}}\left(\Delta X_{1}\right) \text { при } t \geqslant 1,
\end{array}\right.
$$


где $\mathrm{Q}^{\prime c}$ есть абсолютно непрерывная относительно $\mathrm{Q}$ компонента меры $Q^{\prime}$.

Покажем, как тот же ответ следует из формулы (5). Прежде всего, $d \mathrm{P}_{H}^{\prime} / d \mathrm{P}_{H}=1$ и $\sigma=\sigma_{6}=1$, если $\mathrm{Q}=\kappa_{1}$ и $\mathrm{Q}^{\prime}=\kappa_{1}^{\prime}$ сингулярны; если же они несингулярны, что и будет предполагаться в дальнейшем, то $\sigma=\infty$.

Ясно, что $X^{c} \equiv 0$ и $k \equiv 0$, а процессы $N, \mathscr{E}(N)$ и $D$ постоянны при $t<1$ и при $t \geqslant 1$, поэтому достаточно найти их значения в момент $t=1$. Заметим, что в качестве $Y(1, x)$ можно взять $\left(d \mathrm{Q}^{\prime c} / d \mathrm{Q}\right)(x), a_{1}=$ $\mathrm{Q}\left(\mathbf{R}^{d} \backslash\{0\}\right), a_{1}^{\prime}=\mathrm{Q}^{\prime}\left(\mathbf{R}^{d} \backslash\{0\}\right), a_{1,1}^{\prime}=\mathrm{Q}^{\prime c}\left(\mathbf{R}^{d} \backslash\{0\}\right)$.

Разберем 2 случая: $\mathrm{Q}(\{0\})=0$ и $\mathrm{Q}(\{0\})>0$.

Случай 1: $\mathrm{Q}(\{0\})=0$. Очевидно, что

$$
\Delta N_{1}=Y\left(1, \Delta X_{1}\right)-\mathbf{E} Y\left(1, \Delta X_{1}\right)=Y\left(1, \Delta X_{1}\right)-a_{1,1}^{\prime}
$$

и

$$
\mathscr{E}(N)_{1}=1+\Delta N_{1}=1+Y\left(1, \Delta X_{1}\right)-a_{1,1}^{\prime} .
$$

С другой стороны $D_{1}=Y\left(1, \Delta X_{1}\right) /\left(1+Y\left(1, \Delta X_{1}\right)-a_{1,1}^{\prime}\right)$, таким образом $Z_{1}=Y\left(1, \Delta X_{1}\right)$.

В случае локальной абсолютной непрерывности, т.е. в случае $\mathrm{Q}^{\prime} \ll$ $\mathrm{Q}$, имеем $\mathrm{Q}^{\prime}(\{0\})=0, a_{1,1}^{\prime}=a_{1}^{\prime}=1$, т.е. $\mathscr{E}(N)_{1}=Y\left(1, \Delta X_{1}\right)$ и $D_{1}=1$.

Случай 2: $\mathrm{Q}(\{0\})>0$. Имеем

$$
\begin{aligned}
\Delta N_{1}= & {\left[Y\left(1, \Delta X_{1}\right)+\frac{a_{1,1}^{\prime}-1}{1-a_{1}}\right] 1_{\left\{\Delta X_{1} \neq 0\right\}} } \\
& -\mathbf{E}\left(\left[Y\left(1, \Delta X_{1}\right)+\frac{a_{1,1}^{\prime}-1}{1-a_{1}}\right] 1_{\left\{\Delta X_{1} \neq 0\right\}}\right) \\
= & \left(Y\left(1, \Delta X_{1}\right)-1\right) 1_{\left\{\Delta X_{1} \neq 0\right\}}-\frac{a_{1,1}^{\prime}-a_{1}}{1-a_{1}} 1_{\left\{\Delta X_{1}=0\right\}},
\end{aligned}
$$

откуда

$$
\mathscr{E}(N)_{1}=1+\Delta N_{1}=Y\left(1, \Delta X_{1}\right) 1_{\left\{\Delta X_{1} \neq 0\right\}}+\frac{1-a_{1,1}^{\prime}}{1-a_{1}} 1_{\left\{\Delta X_{1}=0\right\}}
$$

С другой стороны, $D_{1}=\left(\left(1-a_{1}^{\prime}\right) /\left(1-a_{1,1}^{\prime}\right)\right) 1_{\left\{\Delta X_{1}=0\right\}}+1_{\left\{\Delta X_{1} \neq 0\right\}}$, откуда

$$
\begin{aligned}
Z_{1} & =Y\left(1, \Delta X_{1}\right) 1_{\left\{\Delta X_{1} \neq 0\right\}}+\frac{1-a_{1}^{\prime}}{1-a_{1}} 1_{\left\{\Delta X_{1}=0\right\}} \\
& =Y\left(1, \Delta X_{1}\right) 1_{\left\{\Delta X_{1} \neq 0\right\}}+\frac{\mathrm{Q}^{\prime}(\{0\})}{\mathrm{Q}(\{0\})} 1_{\left\{\Delta X_{1}=0\right\}}=\frac{d \mathrm{Q}^{\prime}}{d \mathrm{Q}}\left(\Delta X_{1}\right) .
\end{aligned}
$$

В случае локальной абсолютной непрерывности $a_{1}^{\prime}=a_{1,1}^{\prime}$, т.е. $D_{1}=1$. 


\section{2. Доказательство.}

2.1. Сведение к случаю несингулярных мер. Рассмотрим произвольное $t_{0} \in \mathbf{R}_{+}$и обозначим $\mathbf{Q}$ и $\mathbf{Q}^{\prime}$ распределение остановленного процесса $X_{t \wedge t_{0}}$ относительно $\mathrm{P}$ и $\mathrm{P}^{\prime}$ соответственно. Легко видеть, что $\mathrm{Q} \perp \mathrm{Q}^{\prime}$ тогда и только тогда, когда $\mathrm{P}_{t_{0}}^{0} \perp \mathrm{P}_{t_{0}}^{\prime 0}$.

Процесс $X$ является семимартингалом с независимыми приращениями относительно $\mathrm{Q}$ и $\mathrm{Q}^{\prime}$, и версиями его триплетов относительно мер $\mathrm{Q}$ и $\mathrm{Q}^{\prime}$ являются «остановленные» триплеты $\left(B_{t \wedge t_{0}}, C_{t \wedge t_{0}}, 1_{\left[0, t_{0}\right]}(t) \cdot \nu(d t, d x)\right)$, $\left(B_{t \wedge t_{0}}^{\prime}, C_{t \wedge t_{0}}^{\prime}, 1_{\left[0, t_{0}\right]}(t) \cdot \nu^{\prime}(d t, d x)\right)$.

Из критерия сингулярности (см. [1, теорема IV.4.33]) следует, что $\mathrm{P}_{t_{0}}^{0} \not \perp \mathrm{P}_{t_{0}}^{\prime 0}$ при $t_{0}<\sigma$ и $\mathrm{P}_{t_{0}}^{0} \perp \mathrm{P}_{t_{0}}^{\prime 0}$ при $t_{0}>\sigma$. Таким образом, для всех $t_{0}<\sigma$ сужения $\mathrm{P}_{t_{0}}$ и $\mathrm{P}_{t_{0}}^{\prime}$ будут несингулярны, а при всех $t_{0}>\sigma$ сингулярны.

Покажем теперь, что при $t_{0}=\sigma<\infty$ сужения $\mathrm{P}_{t_{0}}$ и $\mathrm{P}_{t_{0}}^{\prime}$ сингулярны. Положим $\mathrm{P}^{*}=\left(\mathrm{P}+\mathrm{P}^{\prime}\right) / 2, Z_{n}=\left.\left(d \mathrm{P} / d \mathrm{P}^{*}\right)\right|_{\mathscr{F}_{\sigma+1 / n}}, Z_{\infty}=\left.\left(d \mathrm{P} / d \mathrm{P}^{*}\right)\right|_{\mathscr{F}_{\sigma}}$.

По теореме Дуба о сходимости обращенных мартингалов $Z_{n} \rightarrow Z_{\infty}$ $\mathrm{P}^{*}$-п.н.

Поскольку сужения $\mathrm{P}_{\sigma+1 / n}$ и $\mathrm{P}_{\sigma+1 / n}^{\prime}$ сингулярны, $Z_{n} \mathrm{P}^{*}$-п.н. может принимать только значения 0 либо 2 , следовательно и $Z_{\infty}$, как предел $Z_{n}$, может принимать только эти два значения, т.е. $\mathrm{P}_{\sigma}$ и $\mathrm{P}_{\sigma}^{\prime}$ сингулярны.

Таким образом, мы доказали формулу (4) и что $Z_{t}=0$ Р-п.н. при $t \geqslant \sigma$.

Пусть теперь $t_{0}<\sigma$, тогда при $t<t_{0}$ процесс $Z$ Р-п.н. совпадает с обобщенным процессом плотности $\mathrm{Q}^{\prime}$ относительно $\mathrm{Q}$. Поскольку процессы $N, \Psi$ и $D$, построенные по парам $\left(\mathrm{P}, \mathrm{P}^{\prime}\right)$ и $\left(\mathrm{Q}, \mathrm{Q}^{\prime}\right)$ совпадают $\mathrm{P}$-п.н. при $t<t_{0}$, то достаточно доказать формулу (5) в случае, когда $\mathrm{P}$ и $\mathrm{P}^{\prime}$ несингулярны на $\mathscr{F}_{\infty}$.

2.2. Построение доминирующей меры. Везде далее мы считаем, что $\mathbf{P} \not \perp \mathbf{P}^{\prime}$. По критерию сингулярности это означает, что $\sigma=\infty$ (в частности $C_{t} \equiv C_{t}^{\prime}$ ) и

$$
h^{0}\left(\frac{1}{2}\right)_{\infty}<\infty .
$$

На следующем шаге доказательства мы построим вероятностную меру $\mathrm{R}$ на $\mathbf{D}\left(\mathbf{R}^{d}\right)$, доминирующую $\mathrm{P}$ и $\mathrm{P}^{\prime}$, относительно которой $X-X_{0}$ будет семимартингалом с независимыми приращениями. В следующих разделах, используя известные выражения для процессов плотности мер $\mathrm{P}$ и $\mathrm{P}^{\prime}$ относительно $\mathrm{R}$, мы найдем явный вид обобщенного процесса плотности $\mathrm{P}^{\prime}$ относительно $\mathrm{P}$. Для упрощения вычислений мера $\mathrm{R}$ будет выбрана таким образом, чтобы процесс плотности $\mathrm{P}$ относительно $\mathrm{R}$ имел максимально простую структуру.

Обозначим $\mathbf{B}=\mathbf{A}^{c}$. Положим

$$
\lambda=1_{J \times \mathbf{R}^{d}} \cdot\left(\frac{\nu+\nu^{\prime}}{2}\right)+1_{J^{c} \times \mathbf{R}^{d}} \cdot\left(\nu+\nu_{2}^{\prime}\right),
$$


тогда $\nu \ll \lambda, \nu^{\prime} \ll \lambda,\left(|x|^{2} \wedge 1\right) * \lambda_{t}<\infty$ для всех $t<\infty, \lambda\left(\{0\} \times \mathbf{R}^{d}\right)=$ $0, \lambda\left(\mathbf{R}_{+} \times\{0\}\right)=0, \lambda\left(\{t\} \times \mathbf{R}^{d}\right) \leqslant 1$ для всех $t<\infty$, и функции $U$, $U^{\prime}$, задаваемые следующими равенствами, будут версиями плотностей $d \nu / d \lambda, d \nu^{\prime} / d \lambda$ соответственно:

$$
\begin{aligned}
& U=1_{\mathbf{A} \cap\left(J^{c} \times \mathbf{R}^{d}\right)}+\frac{2}{1+Y} 1_{\mathbf{A} \cap\left(J \times \mathbf{R}^{d}\right)}, \\
& U^{\prime}=Y 1_{\mathbf{A} \cap\left(J^{c} \times \mathbf{R}^{d}\right)}+\frac{2 Y}{1+Y} 1_{\mathbf{A} \cap\left(J \times \mathbf{R}^{d}\right)}+1_{\mathbf{B} \cap\left(J^{c} \times \mathbf{R}^{d}\right)}+2 \cdot 1_{\mathbf{B} \cap\left(J \times \mathbf{R}^{d}\right)} .
\end{aligned}
$$

Определим триплет

$$
\begin{aligned}
B^{R} & =B+1_{J \times \mathbf{R}^{d}} \hbar *\left(\frac{\nu^{\prime}-\nu}{2}\right)+1_{J^{c} \times \mathbf{R}^{d}} \hbar * \nu_{2}^{\prime}, \\
C^{R} & =C, \\
\nu^{R} & =\lambda
\end{aligned}
$$

и начальное распределение $\mathrm{R}_{H}=\left(\mathrm{P}_{H}+\mathrm{P}_{H}^{\prime}\right) / 2$.

Заметим, что функция $B^{R}$ определена корректно и имеет конечную вариацию на конечных интервалах в силу (6) и $\sigma_{2}=\infty, \Delta B_{t}^{R}=$ $\int \hbar(x) \nu^{R}(\{t\} \times d x)$ для всех $t<\infty$. Обозначим также

$$
\nu_{t}^{R}(d x)=\nu^{R}(\{t\} \times d x), \quad a_{t}^{R}=\nu_{t}^{R}\left(\mathbf{R}^{d}\right) .
$$

Tеорема 2. Существует единственная вероятностная мера $\mathrm{R}$ на $(\Omega, \mathscr{F})$ такая, ито $\mathrm{R}_{0}^{0}=\mathrm{R}_{H}$ и $X-X_{0}$ является семимартингалом с независимьми приращениями относительно $\mathrm{R}$ с триплетом (8). Более того, $\mathrm{P} \ll \mathrm{R}, \mathrm{P}^{\prime} \ll \mathrm{R}$ и процессьи плотности $Z_{R}$ и $Z_{R}^{\prime}$ мер $\mathrm{P}$ и $\mathrm{P}^{\prime}$ соответственно относительно $\mathrm{R}$ имеют вид

$$
\begin{aligned}
Z^{R} & =\frac{d \mathrm{P}_{H}}{d \mathrm{R}_{H}} \mathscr{E}\left(N_{R}\right), \\
Z^{\prime R} & =\frac{d \mathrm{P}_{H}^{\prime}}{d \mathrm{R}_{H}} \mathscr{E}\left(N_{R}^{\prime}\right),
\end{aligned}
$$

где

$$
\begin{aligned}
N^{R} & =\left(U-1+\frac{a-a^{R}}{1-a^{R}} 1_{\left\{a^{R}<1\right\}}\right) *\left(\mu-\nu^{R}\right), \\
N^{\prime R} & =\beta \cdot X^{c}+\left(U^{\prime}-1+\frac{a^{\prime}-a^{R}}{1-a^{R}} 1_{\left\{a^{R}<1\right\}}\right) *\left(\mu-\nu^{R}\right) .
\end{aligned}
$$

3 а м е ч а н и е 3 . Формально процесс $\beta \cdot X^{c}$ в определении $N^{\prime R}$ и процесс $\beta \cdot X^{c}$ в формулировке теоремы 1 имеют разный смысл: в теореме $1 X^{c}=X^{c, P}$ - это непрерывная мартингальная компонента семимартингала $X$ по мере $\mathrm{P}$, а в теореме $2 X^{c}=X^{c, R}-$ непрерывная 
мартингальная компонента семимартингала $X$ по мере R. Кроме того, стохастические интегралы в теоремах 1 и 2 берутся относительно мер P и $\mathrm{R}$ соответственно. Однако, поскольку $\mathrm{P} \ll \mathrm{R}$ и $N^{R}-$ иисто разрывный локальный мартингал (относительно $\mathrm{R}$ ), по теореме Гирсанова $X^{c, R}$ есть локальный мартингал относительно $\mathrm{P}$ и, следовательно, версия процесса $X^{c, P}$. Что касается стохастических интегралов по семимартингалам, а также стохастических экспонент и квадратической вариации, определение которых зависит от вероятностной меры, то мы, не отражая выбор меры в обозначениях, неявно пользуемся тем, что каждый такой объект относительно меры $\mathrm{R}$ является версией соответствующего объекта по мере $\mathrm{P}$ в силу абсолютной непрерывности $\mathrm{P} \ll \mathrm{R}$ (см. [3, гл. $\mathrm{VII}]$ ). Выбор вероятностной меры в стохастических интегралах по компенсированным случайным мерам понятен из контекста. В нескольких случаях, когда это важно, мы используем запись $\stackrel{P}{\text { или }} *$ для обозначения стохастических интегралов по мере $\mathrm{P}$ и $\stackrel{\mathrm{R}}{\mathrm{n}}$ или $\stackrel{\mathrm{R}}{*}$ - для интегралов по мере $\mathrm{R}$.

Д о к а з а т е л ь с т в о. С учетом приведенных выше свойств триплета $\left(B^{R}, C^{R}, \nu^{R}\right)$ существование и единственность меры $\mathrm{R}$ вытекает из $[1$, теорема II.5.2 и следствие II.5.11].

Проверим условия абсолютной непрерывности [1, теорема IV.4.32] для пар $(\mathrm{P}, \mathrm{R})$ и $\left(\mathrm{P}^{\prime}, \mathrm{R}\right)$, где в качестве $(\beta, Y)$ из формулировки упомянутой теоремы в первом случае выступает пара $(0, U)$, во втором - $\left(\beta, U^{\prime}\right)$.

Во-первых, заметим, что $a_{t}^{R}=1$ тогда и только тогда, когда $a_{t}=$ $a_{t}^{\prime}=1$, поскольку случай $a_{t}=0, a_{t}^{\prime}=1$ невозможен в силу того, что $\sigma_{6}=\infty$.

Далее, из (7) видно, что $|U-1| \leqslant\left|U^{\prime}-U\right|$ и $\left|U^{\prime}-1\right| \leqslant\left|U^{\prime}-U\right|$, поэтому $|\hbar(x)(U-1)| * \nu_{t}^{R}<\infty$ и $\left|\hbar(x)\left(U^{\prime}-1\right)\right| * \nu_{t}^{R}<\infty$ для всех $t<\infty$ в силу того, что $\sigma_{2}=\infty$.

Из определений $B^{R}, \nu^{R}=\lambda$, формулы (7), а также формулы (2) и $\sigma_{4}=\infty$ легко проверить, что $B=B^{R}+\hbar(x)(U-1) * \nu^{R}$ и $B^{\prime}=$ $B^{R}+\hbar(x)\left(U^{\prime}-1\right) * \nu^{R}+(c \beta) \cdot A$.

Наконец, заметим, что на множестве $J \times \mathbf{R}^{d}$ справедливо $U+U^{\prime}=2$, поэтому $(\sqrt{U}-1)^{2} \leqslant\left(\sqrt{U}-\sqrt{U^{\prime}}\right)^{2}$ и $\left(\sqrt{U^{\prime}}-1\right)^{2} \leqslant\left(\sqrt{U}-\sqrt{U^{\prime}}\right)^{2} ;$ на множестве $J^{c} \times \mathbf{R}^{d}$ эти два неравенства очевидны из (7).

Аналогично проверяется, что

$$
\sum_{s}\left(\sqrt{1-a_{s}}-\sqrt{1-a_{s}^{R}}\right)^{2} \leqslant \sum_{s}\left(\sqrt{1-a_{s}}-\sqrt{1-a_{s}^{\prime}}\right)^{2}
$$

и

$$
\sum_{s}\left(\sqrt{1-a_{s}^{\prime}}-\sqrt{1-a_{s}^{R}}\right)^{2} \leqslant \sum_{s}\left(\sqrt{1-a_{s}}-\sqrt{1-a_{s}^{\prime}}\right)^{2}
$$


поэтому соотношения

$$
\begin{array}{r}
(\sqrt{U}-1)^{2} * \nu_{\infty}^{R}+\sum_{s}\left(\sqrt{1-a_{s}}-\sqrt{1-a_{s}^{R}}\right)^{2}<\infty \\
\left(\beta^{T} c \beta\right) \cdot A_{\infty}+\left(\sqrt{U^{\prime}}-1\right)^{2} * \nu_{\infty}^{R}+\sum_{s}\left(\sqrt{1-a_{s}^{\prime}}-\sqrt{1-a_{s}^{R}}\right)^{2}<\infty
\end{array}
$$

следуют из (6).

Остальные условия теоремы IV.4.32 [1] очевидно выполнены, и из нее вытекает $\mathrm{P} \ll \mathrm{R}$ и $\mathrm{P}^{\prime} \ll \mathrm{R}$, а представления для плотностей следуют теперь из теоремы III.5.35 [1].

2.3. Вспомогательные вычисления. В этом разделе мы приведем вспомогательные вычисления, результатами которых воспользуемся в следующем разделе для явного выражения обобщенного процесса плотности меры $\mathrm{P}^{\prime}$ относительно $\mathrm{P}$ через их триплеты. Обозначим

$$
\begin{aligned}
W & =U-1+\frac{a-a^{R}}{1-a^{R}} 1_{\left\{a^{R}<1\right\}}, \quad W^{\prime}=U^{\prime}-1+\frac{a^{\prime}-a^{R}}{1-a^{R}} 1_{\left\{a^{R}<1\right\}}, \\
W_{1} & =W 1_{J^{c} \cap J^{\prime}}, \quad W_{2}=W 1_{J^{c} \cap J^{\prime}} .
\end{aligned}
$$

Очевидно, что

$$
\begin{aligned}
\mathscr{E}\left(N^{R}\right) & =\mathscr{E}\left(W_{1} *\left(\mu-\nu^{R}\right)\right) \mathscr{E}\left(W_{2} *\left(\mu-\nu^{R}\right)\right) \mathscr{E}\left(W 1_{J} *\left(\mu-\nu^{R}\right)\right) \\
\mathscr{E}\left(N^{\prime R}\right) & =\mathscr{E}\left(\beta \cdot X^{c}\right) \mathscr{E}\left(W^{\prime} 1_{J^{c}} *\left(\mu-\nu^{R}\right)\right) \mathscr{E}\left(W^{\prime} 1_{J} *\left(\mu-\nu^{R}\right)\right)
\end{aligned}
$$

Обозначим $\mathbf{A}_{s}=\mathbf{A} \cap\left(\{s\} \times \mathbf{R}^{d}\right), \mathbf{B}_{s}=\mathbf{B} \cap\left(\{s\} \times \mathbf{R}^{d}\right)$.

Лемма 1. Пусть $\tau_{1}=\inf \left\{t \in J^{c} \cap J^{c}: \Delta X_{t} \in \mathrm{B}_{t} \backslash\{0\}\right\}$, тогда

$$
\begin{aligned}
& \mathscr{E}\left(W_{1} *\left(\mu-\nu^{R}\right)\right)_{t}=\exp \left(\nu_{2}^{\prime}\left([0, t] \cap J^{c} \cap J^{\prime c}\right)\right) 1_{\llbracket 0, \tau_{1} \llbracket} \quad \mathrm{R}-\text { п.н. } \\
& \mathscr{E}\left(W_{1} *\left(\mu-\nu^{R}\right)\right)_{t}=\exp \left(\nu_{2}^{\prime}\left([0, t] \cap J^{c} \cap J^{\prime c}\right)\right) \quad \text { Р-n.н. }
\end{aligned}
$$

Д о к а з а т е л ь с т в о. Второе соотношение очевидно следует из первого, так как

$$
\mathrm{P}\left(\tau_{1}<\infty\right) \leqslant \mathrm{E} 1_{\mathbf{B}} * \mu_{\infty}=\mathrm{E} 1_{\mathbf{B}} * \nu_{\infty}=0 .
$$

Поскольку $W_{1}=-1_{J^{c} \cap J^{\prime c}} 1_{\mathrm{B}}$ в силу $(7)$, то в силу $(6)$

$$
\left|W_{1}\right| * \nu_{\infty}^{R}=1_{\mathbf{B}} 1_{J^{c} \cap J^{\prime c}}\left(\sqrt{U}-\sqrt{U^{\prime}}\right)^{2} * \nu_{\infty}^{R} \leqslant h^{0}\left(\frac{1}{2}\right)_{\infty}<\infty .
$$

В силу предложения II.1.28 [1] имеем $\left|W_{1}\right| * \mu_{\infty}<\infty$ R-п.н. и

$$
\begin{aligned}
W_{1} *\left(\mu-\nu^{R}\right)_{t} & =W_{1} * \mu_{t}-W_{1} * \nu_{t}^{R} \\
& =\nu_{2}^{\prime}\left([0, t] \cap J^{c} \cap J^{\prime c}\right)-\sum_{s \leqslant t} 1_{\left\{\Delta X_{s} \neq 0\right\}} 1_{J^{c} \cap J^{\prime c}} 1_{\left\{\Delta X_{s} \in \mathbf{B}_{s}\right\}},
\end{aligned}
$$

таким образом

$$
\mathscr{E}\left(W_{1} *\left(\mu-\nu^{R}\right)\right)_{t}=\exp \left(\nu_{2}^{\prime}\left([0, t] \cap J^{c} \cap J^{\prime c}\right)\right) 1_{\llbracket 0, \tau_{1} \llbracket} .
$$


Лемма 2. Пусть $\tau_{2}=\inf \left\{t \in J^{c} \cap J^{\prime}: \Delta X_{t} \in \mathbf{B}_{t} \backslash\{0\}\right\}$, тогда

$$
\begin{aligned}
& \mathscr{E}\left(W_{2} *\left(\mu-\nu^{R}\right)\right)_{t}=1_{\llbracket 0 ; \tau_{2} \llbracket} \prod_{\left\{(\omega, s): s \leqslant t, s \in J^{c} \cap J^{\prime}, \Delta X_{s}=0\right\}} \frac{1}{1-a_{s}^{\prime}} \quad \text { R-n.н. } \\
& \mathscr{E}\left(W_{2} *\left(\mu-\nu^{R}\right)\right)_{t}=\prod_{\left\{(\omega, s): s \leqslant t, s \in J^{c} \cap J^{\prime}, \Delta X_{s}=0\right\}} \frac{1}{1-a_{s}^{\prime}} \quad \text { P-n.н. }
\end{aligned}
$$

Д о к а з а т е л ь с т в о. Второе утверждение следует из первого также как и в предыдущей лемме. Поскольку $J^{c} \cap J^{\prime} \subseteq\left\{t: a_{t}=0, a_{t}^{R}=\right.$ $\left.a_{t}^{\prime}<1\right\}$, из (7) следует, что

$$
W_{2}=-1_{J^{c} \cap J^{\prime}}\left(1_{\mathbf{B}}+\frac{a^{\prime}}{1-a^{\prime}}\right)=-1_{J^{c} \cap J^{\prime}} \frac{1}{1-a^{\prime}} \quad \nu^{R} \text {-П.в. }
$$

Далее, для $t \in J^{c} \cap J^{\prime}$ имеем $a_{t}^{\prime}=\int 1_{\mathbf{B}_{t}} \nu_{t}^{R}(d x)=\int 1_{\mathbf{B}_{t}}(\sqrt{U(t, x)}-$ $\left.\sqrt{U^{\prime}(t, x)}\right)^{2} \nu_{t}^{R}(d x)$, поэтому из (6) следует, что $\sum_{s \in J^{c} \cap J^{\prime}} a_{s}^{\prime}<\infty$, откуда вытекает сходимость ряда $\sum_{s \in J^{c} \cap J^{\prime}} \frac{a_{s}^{\prime}}{1-a_{s}^{\prime}}$, значит

$$
\left|W_{2}\right| * \nu_{\infty}^{R}=\sum_{s \in J^{c} \cap J^{\prime}} \frac{a_{s}^{\prime}}{1-a_{s}^{\prime}}<\infty
$$

и по [1, предложение II.1.28]

$$
\begin{aligned}
W_{2} *\left(\mu-\nu^{R}\right)_{t} & =W_{2} * \mu_{t}-W_{2} * \nu_{t}^{R} \\
& =-\sum_{\substack{s \leqslant t \\
s \in J \subset J^{\prime}}} 1_{\left\{\Delta X_{s} \neq 0\right\}} 1_{\left\{\Delta X_{s} \in \mathbf{B}_{s}\right\}}+\sum_{\substack{s \leq t, s \in J^{\prime} \cap J^{\prime}}} \frac{a_{s}^{\prime}}{1-a_{s}^{\prime}} 1_{\left\{\Delta X_{s}=0\right\}}
\end{aligned}
$$

и

$$
\mathscr{E}\left(W_{2} *\left(\mu-\nu^{R}\right)\right)_{t}=1_{\llbracket 0 ; \tau_{2} \llbracket} \prod_{\{(\omega, s): s \leqslant t,} \prod_{\left.s \in J^{c} \cap J^{\prime}, \Delta X_{s}=0\right\}} \frac{1}{1-a_{s}^{\prime}} .
$$

Лемма 3. Прочесс $M=W^{\prime} 1_{J^{c}} \stackrel{\mathrm{R}}{*}\left(\mu-\nu^{R}\right)$ является локальныл мартингалом по мере $\mathrm{P}$ и является версией стохастического интеграла $\widetilde{M}=W^{\prime} 1_{J^{c}} \stackrel{\mathrm{P}}{*}(\mu-\nu)$, в частности $W^{\prime} 1_{J^{c}} \in G_{l o c}(\mu, \mathrm{P})$.

Д о к а з а т е л ь с т в о. Пусть $t \in J^{c}$. Тогда $a_{t}^{R}=a_{t}^{\prime}<1$, поэтому

$$
W^{\prime} 1_{J^{c}}=\left(U^{\prime}-1\right) 1_{J^{c}}=(Y-1) 1_{J^{c}} 1_{\mathbf{A}} .
$$

Более того, $\int W^{\prime}(t, x) \nu_{t}^{R}(d x)=\int\left(U^{\prime}(t, x)-1\right) \nu_{t}^{R}(d x)=a_{t}^{\prime}-a_{t}^{R}=0$, поэтому

$$
\Delta M_{s}=\left(Y\left(s, \Delta X_{s}\right)-1\right) 1_{J^{c}}(s) 1_{\left\{\Delta X_{s} \in \mathbf{A}_{s} \backslash\{0\}\right\}} .
$$

С другой стороны, в силу (9) имеем $\Delta Z^{R} 1_{J^{c}}=Z_{-}^{R} \Delta N^{R} 1_{J^{c}}$, а $\Delta N^{R} 1_{J^{c}}=\Delta\left(W_{1} *\left(\mu-\nu^{R}\right)\right)+\Delta\left(W_{2} *\left(\mu-\nu^{R}\right)\right)$. 
Из явного вида процессов $W_{1} *\left(\mu-\nu^{R}\right)$ и $W_{2} *\left(\mu-\nu^{R}\right)$, указанных в доказательствах лемм 1 и 2 , следует, что $\Delta M \Delta Z^{R}=0$ и $\left[M, Z^{R}\right]=0$, так как $M$ и $Z^{R}$ - чисто разрывные локальные мартингалы относительно $\mathrm{R}$.

По теореме Гирсанова (см. [3, теорема 7.25]) $M$ является чисто разрывным локальным мартингалом по мере $\mathrm{P}$, в частности, процесс $\left[\sum_{s \leqslant .}\left(\Delta M_{s}\right)^{2}\right]^{1 / 2}$ является локально интегрируемым по мере $\mathrm{P}$.

Поскольку $a_{t}=0$, и значит $\int W^{\prime}(t, x) \nu_{t}(d x)=0$, для $t \in J^{c}$, процесс $\widetilde{M}$, если $W^{\prime} 1_{J^{c}} \in G_{\text {loc }}(\mu, \mathrm{P})$, есть чисто разрывный локальный мартингал по мере $\mathrm{P}$ со скачками $V_{s}:=W^{\prime}\left(s, \Delta X_{s}\right) 1_{\left\{X_{s} \neq 0\right\}} 1_{J^{c}}(s)=\Delta M_{s}$, а условие $W^{\prime} 1_{J^{c}} \in G_{\text {loc }}(\mu, \mathrm{P})$ означает, что $\left[\sum_{s \leqslant} \cdot V_{s}^{2}\right]^{1 / 2}$ является локально интегрируемым по мере Р. Таким образом, последнее условие выполнено и $\Delta M=\Delta \widetilde{M}$, откуда следует последнее утверждение леммы.

Лемма 4. Р-п.н. справедливо

$$
\frac{\mathscr{E}\left(W^{\prime} 1_{J} *\left(\mu-\nu^{R}\right)\right)}{\mathscr{E}\left(W 1_{J} *\left(\mu-\nu^{R}\right)\right)}=\mathscr{E}(L-\Phi)=\mathscr{E}(L) \Upsilon
$$

гдe

$$
\begin{aligned}
& L=K *(\mu-\nu), \quad K=(Y-1) 1_{J} 1_{\mathbf{A}}+1_{J} \frac{a_{1}^{\prime}-a}{1-a} 1_{\{a<1\}}, \\
& \Phi=\sum_{\substack{s \leqslant, \Delta X_{s}=0}} \frac{a_{2, s}^{\prime}}{1-a_{s}}+\sum_{s \leqslant \cdot, a_{s}=1}\left(1-a_{1, s}^{\prime}\right) \text {, } \\
& \Upsilon=\prod_{\substack{s \leqslant ., \Delta<a_{s}<1, \Delta X_{s}=0, a_{1, s}^{\prime}<1}} \frac{1-a_{s}^{\prime}}{1-a_{1, s}^{\prime}} \prod_{\substack{s \leqslant, a_{s}=1, a_{1, s}^{\prime}<1}} \frac{Y\left(s, \Delta X_{s}\right)}{1+Y\left(s, \Delta X_{s}\right)-a_{1, s}^{\prime}} \text {. }
\end{aligned}
$$

Д о к а з а т е л ь с т в о. Проверим, что процессы $L, \Phi, \Upsilon$ определены корректно и имеет место второе равенство в соотношении (15).

Во-первых,

$$
\begin{aligned}
\mathrm{E} \Phi_{\infty} & =\sum_{s: 0<a_{s}<1} \frac{a_{2, s}^{\prime}}{1-a_{s}} \mathrm{P}\left\{\Delta X_{s}=0\right\}+\sum_{s: a_{s}=1}\left(1-a_{1, s}^{\prime}\right) \\
& =\sum_{s: 0<a_{s}<1} a_{2, s}^{\prime}+\sum_{s: a_{s}=1}\left(1-a_{1, s}^{\prime}\right)=\sum_{s: a_{s}>0} a_{2, s}^{\prime}+\sum_{s: a_{s}=1}\left(1-a_{s}^{\prime}\right) \\
& =\left(\sqrt{U}-\sqrt{U^{\prime}}\right)^{2} 1_{\mathbf{B}} 1_{J} * \nu_{\infty}^{R}+\sum_{s: a_{s}=1}\left(\sqrt{1-a_{s}}-\sqrt{1-a_{s}^{\prime}}\right)^{2} \\
& \leqslant 2 h^{0}\left(\frac{1}{2}\right)_{\infty}<\infty
\end{aligned}
$$

в силу (6).

Обозначим $\widehat{K}_{t}=\int K(t, x) \nu_{t}(d x)$, тогда

$$
\widehat{K}_{s}=\left(a_{1, s}^{\prime}-a_{s}\right) 1_{\left\{a_{s}=1\right\}}+\frac{a_{1, s}^{\prime}-a_{s}}{1-a_{s}} 1_{\left\{0<a_{s}<1\right\}} .
$$


Далее,

$$
\begin{aligned}
\widetilde{K}_{s}:= & K\left(s, \Delta X_{s}\right) 1_{\left\{\Delta X_{s} \neq 0\right\}}-\widehat{K}_{s} \\
= & \left(Y\left(s, \Delta X_{s}\right)-1\right) 1_{J} 1_{\left\{\Delta X_{s} \in \mathbf{A}_{s}\right\}} 1_{\left\{\Delta X_{s} \neq 0\right\}} \\
& -\frac{a_{1, s}^{\prime}-a_{s}}{1-a_{s}} 1_{\left\{0<a_{s}<1\right\}} 1_{\left\{\Delta X_{s}=0\right\}}+\left(1-a_{1, s}^{\prime}\right) 1_{\left\{a_{s}=1\right\}} .
\end{aligned}
$$

Из последней формулы легко видеть, что

$$
\widetilde{K} \geqslant-1 \quad \text { и } \quad\{\widetilde{K}=-1\} \subseteq\{\Delta \Phi=0\} .
$$

Покажем, что $C^{\prime}(K)_{\infty} \leqslant 2 h^{0}(1 / 2)_{\infty}<\infty$, где

$$
C^{\prime}(K)_{t}:=(1-\sqrt{1+K-\widehat{K}})^{2} * \nu_{t}+\sum_{s \leqslant t}\left(1-a_{s}\right)\left(1-\sqrt{1-\widehat{K}_{s}}\right)^{2} .
$$

Действительно, поскольку $K=K 1_{J}$,

$$
\begin{aligned}
C^{\prime}(K)_{\infty}= & \sum_{s: a_{s}>0}\left(\int\left(1-\sqrt{1+K_{s}-\widehat{K}_{s}}\right)^{2} d \nu_{s}\right. \\
& +\sum_{s: 0<a_{s}<1}\left(1-a_{s}\right)\left(1-\sqrt{1-\widehat{K}_{s}}\right)^{2} \\
= & \sum_{s: a_{s}=1} \int\left(1-\sqrt{Y_{s}+1-a_{1, s}^{\prime}}\right)^{2} d \nu_{s} \\
& +\sum_{s: 0<a_{s}<1}\left(\int\left(1-\sqrt{Y_{s}}\right)^{2} d \nu_{s}+\left(\sqrt{1-a_{s}}-\sqrt{1-a_{1, s}^{\prime}}\right)^{2}\right) \\
\leqslant & \sum_{s: a_{s}=1}\left(\int\left(1+Y_{s}-2 \sqrt{Y_{s}}\right) d \nu_{s}+1-a_{1, s}^{\prime}\right) \\
& +\sum_{s: 0<a_{s}<1}\left(\int\left(1-\sqrt{Y_{s}}\right)^{2} d \nu_{s}+a_{2, s}^{\prime}+1-a_{s}+1-a_{s}^{\prime}\right. \\
= & \sum_{s: a_{s}>0} \int\left(\sqrt{U_{s}}-\sqrt{U_{s}^{\prime}}\right)^{2} d \nu_{s}^{R}+\sum_{s: a_{s}>0}\left(\sqrt{1-a_{s}}-\sqrt{1-a_{s}^{\prime}}\right)^{2} \\
\leqslant & 2 h^{0}\left(\frac{1}{2}\right)_{\infty} \cdot
\end{aligned}
$$

Таким образом, в силу утверждения d) Теоремы II.1.33 из [1], $K \in G_{\text {lос }}(\mu, \mathrm{P})$, значит $L$ корректно определен и является локальным мартингалом, причем $\Delta L=\widetilde{K}$.

Далее положим $\widetilde{\Phi}=\left(1_{\{\Delta L>-1\}} /(1+\Delta L)\right) \cdot \Phi$. Очевидно, что $\widetilde{\Phi}-$ возрастающий процесс с конечными значениями и

$$
L-\widetilde{\Phi}-[L, \widetilde{\Phi}]=L-(1+\Delta L) \cdot \widetilde{\Phi}=L-1_{\{\Delta L>-1\}} \cdot \Phi=L-\Phi,
$$


где последнее равенство вытекает из (16).

Таким образом, по формуле Йора $\mathscr{E}(L) \mathscr{E}(-\widetilde{\Phi})=\mathscr{E}(L-\Phi)$.

Учитывая, что $\mathrm{P}\left\{\Delta X_{s}=0, a_{s}=1\right\}=0$, непосредственным подсчетом проверяется, что $\Upsilon=\mathscr{E}(-\widetilde{\Phi})$. Значит процесс $\Upsilon$ корректно определен и имеет место второе равенство в формуле (15).

Перейдем теперь к проверке первого равенства в (15). Заметим, что процесс плотности меры $\mathrm{P}$ относительно $\mathrm{R}$ строго положителен $\mathrm{P}$-п.н., поэтому знаменатель в левой части (15) не обращается в нуль Р-п.н., и достаточно проверить, что $\mathscr{E}\left(M^{1}\right)=\mathscr{E}\left(M^{2}\right) \mathscr{E}\left(M^{3}\right)$, или эквивалентно, в силу формулы Йора, что

$$
M^{1}=M^{2}+M^{3}+\left[M^{2}, M^{3}\right],
$$

где

$$
M^{1}=W^{\prime} 1_{J} *\left(\mu-\nu^{R}\right), \quad M^{2}=W 1_{J} *\left(\mu-\nu^{R}\right), \quad M^{3}=L-\Phi .
$$

Процессы $M^{i}, i=1,2$, являются локальными мартингалами по мере $\mathrm{R}$, причем $1_{J} \stackrel{\mathrm{R}}{\cdot} M^{i}=M^{i}, i=1,2$. Из общей теории замены меры (см. [3, гл. VII]) известно, что $M^{i}, i=1,2$, являются семимартингалами по мере $\mathrm{P}$, причем процессы $M^{i}=1_{J} \stackrel{\mathrm{R}}{\cdot} M^{i}$ являются Р-версиями стохастических интегралов $1_{J} \stackrel{\mathrm{P}}{ } \cdot M^{i}, i=1,2$. Кроме того, $M^{3}=1_{J} \stackrel{\mathrm{P}}{\mathrm{P}} M^{3} \mathrm{P}$-п.н.

Пусть $J=\left\{t_{1}, \ldots, t_{n}, \ldots\right\}$. Из общих свойств стохастического интеграла следует, что для любого $t<\infty$

$$
1_{J} \stackrel{\mathrm{P}}{\cdot} M_{t}^{i}=\mathrm{P}-\lim _{k \rightarrow \infty} \sum_{j \leqslant k, t_{j} \leqslant t} \Delta M_{t_{j}}^{i}, \quad i=1,2,3,
$$

где предел понимается в смысле сходимости по вероятности относительно Р. Таким образом, для доказательства соотношения (17) достаточно проверить, что $\Delta M^{1}=\Delta M^{2}+\Delta M^{3}+\Delta M^{2} \Delta M^{3}$ Р-п.н.

Заметим, что $W^{\prime} 1_{J}=-W 1_{J}$, поэтому $\Delta M^{1}=-\Delta M^{2}$.

Пусть $s \in J$. Поскольку $\mathrm{P}\left\{\Delta X_{s} \in \mathbf{B}_{s} \backslash\{0\}\right\}=0$, имеем

$$
\begin{aligned}
\Delta M_{s}^{2}= & \frac{1-Y\left(s, \Delta X_{s}\right)}{1+Y\left(s, \Delta X_{s}\right)} 1_{\left\{\Delta X_{s} \in \mathbf{A}_{s}\right\}} 1_{\left\{\Delta X_{s} \neq 0\right\}} 1_{J} \\
& -1_{\left\{\Delta X_{s}=0\right\}} \frac{a_{s}-a_{s}^{\prime}}{2-a_{s}-a_{s}^{\prime}} 1_{\left\{a_{s} a_{s}^{\prime}<1\right\} \cap J} \quad \text { P-п.н., } \\
\Delta M_{s}^{3}=\widetilde{K}_{s}-\Delta \Phi_{s}= & \left(Y\left(s, \Delta X_{s}\right)-1\right) 1_{J} 1_{\left\{\Delta X_{s} \neq 0\right\}} 1_{\left\{\Delta X_{s} \in \mathbf{A}_{s}\right\}} \\
& -\frac{a_{s}^{\prime}-a_{s}}{1-a_{s}} 1_{\left\{\Delta X_{s}=0\right\}} 1_{\left\{0<a_{s}<1\right\}},
\end{aligned}
$$

откуда, поскольку $\mathrm{P}\left\{\Delta X_{s}=0, a_{s}=1\right\}=0$, непосредственным вычислением проверяется, что $2 \Delta M^{2}+\Delta M^{3}+\Delta M^{2} \Delta M^{3}=0$ Р-п.н., что влечет выполнение (17) и справедливость первого соотношения в формуле (15). 
2.4. Завершение доказательства теоремы 1. С учетом формул (9)-(12) и результатов лемм 1-4 мы получаем следующее представление для обобщенного процесса плотности меры $\mathrm{P}^{\prime}$ относительно $\mathrm{P}$ (в предположении, что $\left.\mathrm{P} \not \perp \mathrm{P}^{\prime}\right)$ : Р-п.н.

$$
Z=\frac{Z^{\prime} R}{Z^{R}}=\frac{d \mathrm{P}_{H}^{\prime}}{d \mathrm{P}_{H}} e^{-k} \mathscr{E}\left(\beta \cdot X^{c}\right) \mathscr{E}(G) \mathscr{E}(L) \Upsilon \Lambda,
$$

где $G=W^{\prime} 1_{J^{c}} *(\mu-\nu)$ (см. лемму 3$)$ и $\Lambda_{t}=\prod_{\substack{s \leqslant t, s \in J^{c} J^{\prime} \\ \Delta X_{s}=0}}\left(1-a_{s}^{\prime}\right)$ (см. лемму 2). Заметим, что поскольку $\nu(\mathbf{B})=0$, в определении процесса $N$ можно заменить $Y-1$ на $(Y-1) 1_{\mathbf{A}}$. Отсюда, учитывая очевидное соотношение

$$
\left\{s: a_{s}=0\right\} \subseteq\left\{s: a_{1, s}^{\prime}=0\right\}
$$

и равенства (13), получаем

$$
N=\beta \cdot X^{c}+G+L,
$$

причем $\mathscr{E}(N)$ разбивается в произведение стохастических экспонент слагаемых в правой части (20). Для доказательства первого представления из формулы (5) остается заметить, что с учетом (19)

$$
D=e^{-k} \Upsilon \Lambda
$$

Заметим, что $\Lambda=\mathscr{E}(-\Gamma)$, где $\Gamma_{t}=\sum_{\substack{s \leqslant t, s \in J^{c} \cap J^{\prime} \\ \Delta X_{s}=0}} a_{s}^{\prime}$. С учетом второго равенства в (15) это позволяет переписать соотношение (18) в виде

$$
Z=\frac{d \mathrm{P}_{H}^{\prime}}{d \mathrm{P}_{H}} e^{-k} \mathscr{E}\left(\beta \cdot X^{c}\right) \mathscr{E}(G) \mathscr{E}(L-\Phi) \mathscr{E}(-\Gamma) .
$$

Поскольку процессы $G, L-\Phi$ и $\Gamma$ не имеют попарно одновременных скачков (для пары $G$ и $\Gamma$ это следует из формулы (14)), а процессы $k$ и $\beta \cdot X^{c}$ непрерывны, произведение всех экспонент в (21) представляется как стохастическая экспонента суммы их аргументов. Поэтому второе представление в формуле (5) следует из (20) и соотношения $k+\Phi+\Gamma=\Psi$, которое легко проверяется с учетом (19).

\section{СПИСОК ЛИТЕРАТУРЫ}

1. Жакод Ж., Ширяев А.Н. Предельные теоремы для случайных процессов. Т. І. М.: Физматлит, 1994, 544 с.

2. Sato K. Density transformation in Lévy processes. Lecture notes for «Concentrated Advanced Course on Lévy Processes». MaPhySto, Centre for Mathematical Physics and Stochastics, Department of Mathematical Sciences, University of Aarhus, 2000.

3. Jacod J. Calcul shochastique et problèmes de martingales. Berlin, Heidelberg, New York: Springer-Verlag, 1979. 